\title{
An effective method for monitoring the vibration data of bearings to diagnose and minimize defects
}

\author{
$Y C \mathrm{Chu}^{1}, T N \mathrm{Pham}^{1,2}, F R \mathrm{Hsu}^{1}, M J \mathrm{Tuw}^{1}, C W \mathrm{Tan}^{1}, M C \mathrm{Chay}^{1}, S C \mathrm{Lim}^{1}$, and $M F$ \\ $\mathrm{Tsai}^{3, *}$ \\ ${ }^{1}$ Department of Information Engineering and Computer Science, Feng Chia University, Taiwan, \\ R.O.C. \\ ${ }^{2}$ Department of Electronics and Communications Technology, Thai Nguyen University of \\ Information and Communication Technology, Vietnam. \\ ${ }^{3}$ Department of Electronic Engineering, National United University, Taiwan, R.O.C.
}

\begin{abstract}
Monitoring of vibration in machine tools is becoming a very important application in industry to reduce machine failures, maintenance costs, and dead time. In this paper, we propose a method to identify possible faults based on vibration data from which predictions about the working condition of the machine tools can be made. We used an accelerometer to collect the vibration data from which to analyse the health of machine tools by diagnosing whether they are in good or faulty condition for working. In our experiments, we introduced a machine called the Reliance Electric motor, which has a bearing running inside it. Our research analyses vibration data from components of the bearing including the outer bearing, inner bearing, and rolling element. The experimental results show that our method is highly accurate in diagnosing failures and significantly reduces the maintenance costs of machine tools.
\end{abstract}

\section{Introduction}

Since ancient times, one of the most important manufacturers has been the machine. Once a machine beings to malfunction or is damaged, it is likely to lead to operating loss for the manufacturer. Some machines suffer parts failure and a fault will not be noticed immediately. Therefore, in order to avoid the abnormal situation remaining undetected for a long time, these failures should be diagnosed and repaired immediately to avoid many follow-up problems. Today, vibration analysis techniques [1-5] are commonly used to test the operating conditions of machine tools and to diagnose deterioration so as to minimize maintenance costs as well as machine down time. In particular, Senthilkumar et al. [1] focus on the analysis of the characteristic frequencies in the vibration data of tapered roller bearings, gear mesh, and belt drivers to find the source of vibration, thus allowing correct and accurate predictions of defects to be made. The authors of studies [3-5] focus extensively on the analysis of vibration data of ball bearings to provide diagnosis of failures.

\footnotetext{
* Corresponding author: mingfongtsai@gmail.com
} 
These techniques are applied to collect data about the vibration of the machine tools to the computer and then to analyse and measure the damage that corresponds to the characteristics of the vibration data. To accomplish this, Fourier transform (FT) from the time domain to the frequency domain is applied to the collected signals to facilitate statistical observation. Therefore, our motivation and goals are based on existing vibration analysis techniques combined with software tools to provide effective predictions of possible failures during the operation of the machine. In our study, we focused on analysing the vibration data of a bearing. Fast Fourier Transform (FFT) and vibration analysis will be applied to the data captured and the existing software R-Studio will be used to write a program that identifies whether a bearing is operating abnormally or not.

In industry, in order to create wisdom machines, failures need to be monitored, diagnosed, and corrected in time. In this study, using spread spectrum data obtained after Fourier transform, we set up a fault classification model which provides diagnostics and detection of abnormalities of machine tools. Our main goals in this research are to reduce failures and human labour costs, improve productivity and efficiency, and enhance competitiveness.

To achieve these goals, our study carries out the analysis and application of the following techniques:

- The use of R-Studio software for collection and analysis of vibration data.

- Fourier transform to analyse the vibration data.

- Modelling of failures based on the spread spectrum to find the peak and valley faults, from which the limits of amplitude to realize each type of failure are found. The modelling method is one of our main contributions in this study. The classification of types of errors enables us to easily detect and diagnose whether the machine is operating in good condition or abnormally. This paper is organized as follows: Section 2 describes the proposed method using in our paper, Section 3 discusses the experimental results and the implementations of our proposed method, and Section 4 presents the conclusions.

\section{Proposed Method}

\subsection{Data analysis}

Fig. 1. System flow processes.

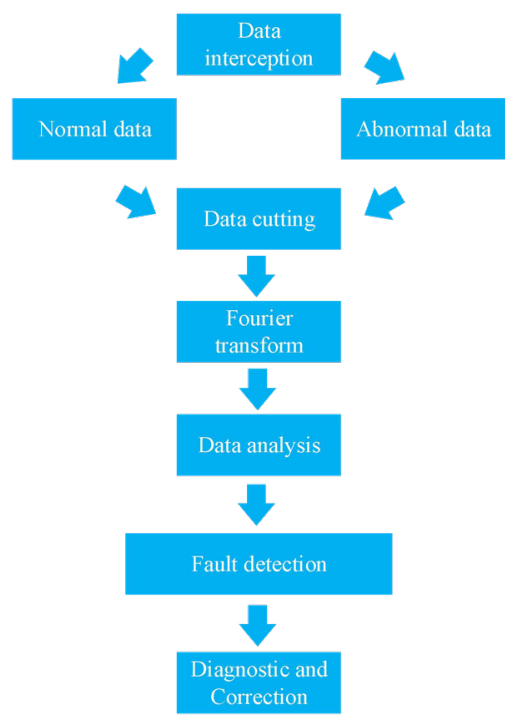


In our study, we used a specific machine with an SKF6205 drive end bearing and F6203 fan end bearing. The main shaft is used for fault detection and analysis. The vibration data are taken from the upper part of the motor fan and the bearing of the drive end, and the acceleration signal has a frequency of $12,000 /$ second. After intercepting known failures and known normal vibration data, the data are processed. As shown in figure 1, from the first steps, the data collected including normal and abnormal data will be analysed to diagnose failure. The collected data will be cut into time intervals corresponding to a certain number of records. The next steps involve applying Fourier transform from the time domain to the frequency domain to these data in order to observe and evaluate whether failure has occurred. After the Fourier transform, the data can be classified based on their peak coordinates as shown in Section 3.1. After making predictions of possible failures, the final step is to carry out repairs and maintenance on time. Detailed algorithms for classification and fault diagnosis are presented in Section 3.3. Since this study mainly focuses on a particular machine spindle, it encounters fewer special cases and therefore does not need to be applied to other more complex algorithms. Therefore, our research only focuses on some components of the machine and does not necessarily apply to all spindle fault detection.

\subsection{Statistical analysis}

The vibration of the machine tool is measured in different locations: the outer race, inner race, and rolling (ball) element. These locations experience more vibration than other places. The frequency spectrum is shown in figure 2 below. After Fourier transform, we obtain the coordinates of each peak corresponding to frequencies. In this case, when Fourier transform has been applied to the normal and abnormal data, the peak points corresponding to the coordinates $\mathrm{P}(\mathrm{x}, \mathrm{y})$ are as follows: for the normal data, $\mathrm{P}_{1}(2073,10.93092)$, as shown in figure 2 (a); $\mathrm{P}_{2}(7175,55.02376)$, as shown in figure $2(\mathrm{~b}) ; \mathrm{P}_{3}(6463,3.909882)$, as shown in figure 2 (c); $\mathrm{P}_{4}(5269,310.4004)$, as shown in figure $2(\mathrm{~d}) ; \mathrm{P}_{5}(6891,245.6510)$, as shown in figure $2(\mathrm{e})$; and $\mathrm{P}_{6}(6739,16.67169)$, as shown in figure 2 (f).

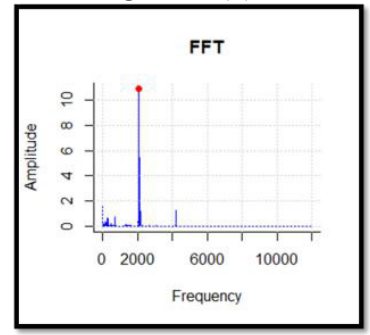

(a) Normal data

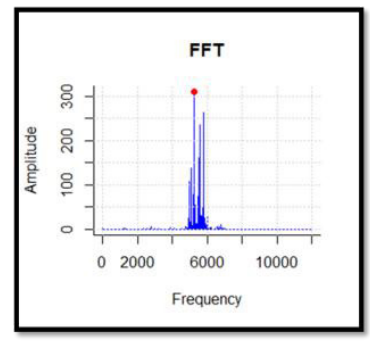

(d) Outer bearing damage (3 o'clock direction)

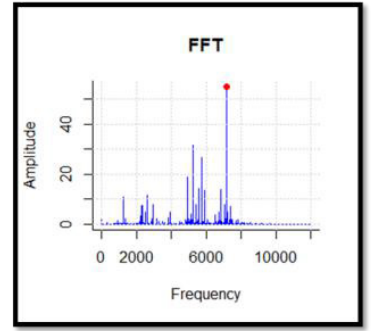

(b) Inner bearing damage

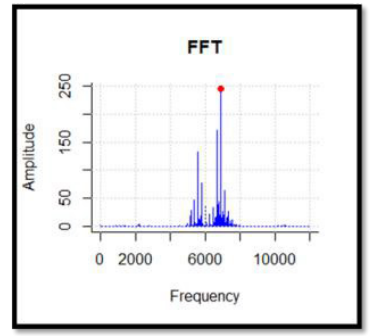

(e) Outer bearing damage (6 o'clock direction)

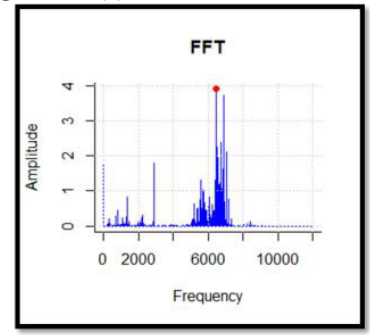

(c) Ball damage

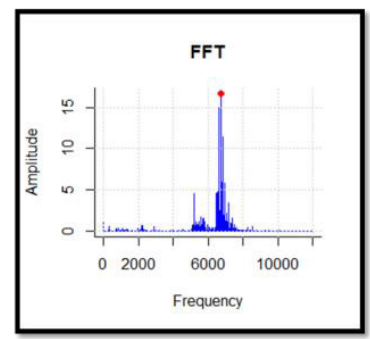

(f) Outer bearing damage (12 o'clock direction)

Fig. 2. Frequency spectra of vibration data after Fourier transform: (a) normal data; (b) inner bearing damage; (c) ball damage; (d) outer bearing damage (3 o'clock direction); (e) outer bearing damage (6 o'clock direction); (f) outer bearing damage (12 o'clock direction). 
The experimental data are divided into six different types comprising normal values and five different types of fault values. Figure 3 and table 1 show statistical representations of the coordinates of the peaks of these six different data types. Table 1 explains the different types of faults that are shown in figure 3 .

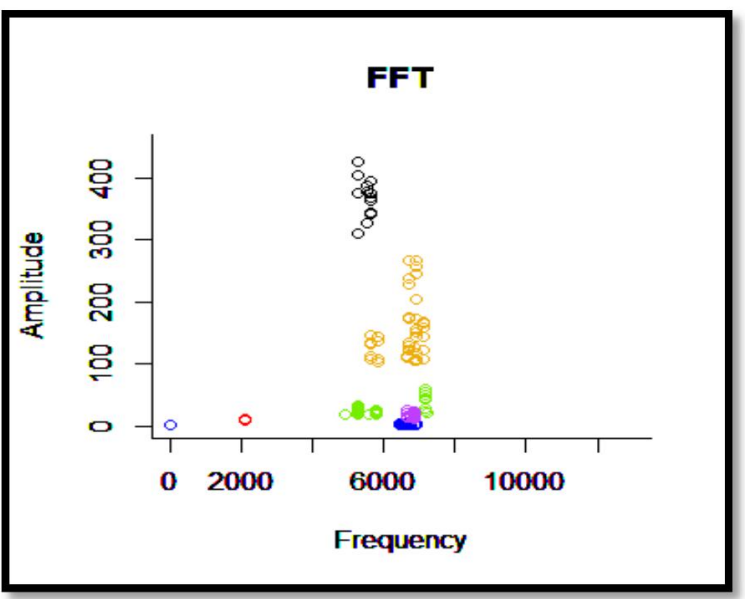

Fig. 3. Fault peak statistics.

Table 1. Fault type description.

\begin{tabular}{|l|l|}
\hline Colour Representation & Fault Type \\
\hline Red & Normal value \\
\hline Blue & Inner bearing damage \\
\hline Green & Ball damage \\
\hline Black & Outer bearing damage (3 o'clock direction) \\
\hline Yellow & Outer bearing damage (6 o'clock direction) \\
\hline Violet & Outer bearing damage (12 o'clock direction) \\
\hline
\end{tabular}

\section{Experimental Design}

\subsection{Fault Features}

Table 2. Limit values for different types of failures.

\begin{tabular}{|c|c|c|c|c|}
\hline Types of & $\begin{array}{c}\mathrm{X} \\
\text { (minimum) }\end{array}$ & $\begin{array}{c}\mathrm{X} \\
\text { (maximum) }\end{array}$ & $\begin{array}{c}\mathrm{Y} \\
\text { (minimum) }\end{array}$ & $\begin{array}{c}\mathrm{Y} \\
\text { (maximum) }\end{array}$ \\
\hline Normal value & 2073 & 2073 & 9.49 & 11.49 \\
\hline Inner bearing damage & 4911 & 7211 & 20.04 & 67.44 \\
\hline Ball damage & 6463 & 6895 & 2.50 & 5.16 \\
\hline $\begin{array}{c}\text { Outer bearing damage } \\
\text { (3 o'clock direction) }\end{array}$ & 5269 & 5605 & 300.55 & 495.16 \\
\hline $\begin{array}{c}\text { Outer bearing damage } \\
\text { (6 o'clock direction) }\end{array}$ & 5599 & 7107 & 102.60 & 267.78 \\
\hline $\begin{array}{c}\text { Outer bearing damage } \\
\text { (12 o'clock direction) }\end{array}$ & 6619 & 6859 & 11.44 & 27.53 \\
\hline
\end{tabular}

From the analysis above, we can find the maximum and minimum of each type of data as our modelling method. Table 2 shows a statistical representation of the range of coordinates 
of each type of data. In this table, we can see that the normal value is fixed at 2073 on the $\mathrm{x}$-axis and between 9.49 and 11.49 on the $\mathrm{y}$-axis. From this set of data, we can also observe that ball damage, outer bearing damage (3 o'clock direction), outer bearing damage (6 o'clock direction), and outer bearing damage (12 o'clock direction) fall in the range of the $x-$ coordinate of inner bearing damage from the minimum to the maximum values of the $\mathrm{x}$ axis. However, the difference in their y coordinates is quite large. From this statistical data, we can set up a model to determine the type of failure of the data obtained.

\subsection{Modelling Method}

From the failure identification table, we obtained a model for the classification of different types of data. Although this method may seem crude, such an approach would allow us to change information at any time and improve the success rate of the result obtained. The modelling process is performed according to the following steps: first, find the maximum and minimum values of the horizontal and vertical axes of the data from the analysed peak value; second, create Excel files manually; and third, store the horizontal and vertical axes values. In manually creating a file, we only need to input the observed feature value into the Excel file and use our program to determine the value of the file in order to determine the result of the failure type. From our previous feature table, the minimum frequency value is input in the upper left corner, the maximum frequency value in the lower left corner, the minimum amplitude in the upper right corner, and the maximum amplitude in the lower right corner; then our program will return the result of this type of failure. We only need to compare the position of the test data with the statistical data to see whether the test data fall within which range and shown in figure 4.

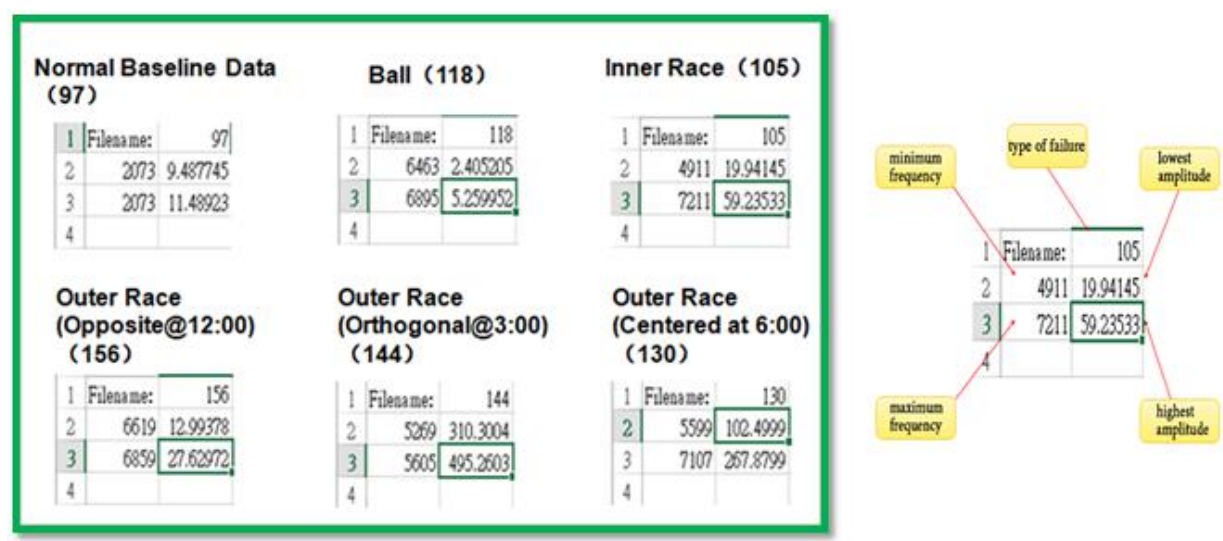

Fig. 4. Excel file modelling and an example description.

\subsection{Experimental Results}

Table 3. Data test.

\begin{tabular}{|c|c|c|}
\hline \multicolumn{2}{|c|}{ Failure test (seconds) } & Success rate (\%) \\
\hline $0.25(3000$ records $)$ & $0.75(9000$ records $)$ & 42.86 \\
\hline $0.16(2000$ records $)$ & $0.84(10000$ records $)$ & 57.14 \\
\hline & $1.00(12000$ records $)$ & 96.00 \\
\hline
\end{tabular}


Combining the above analysis methods, we use a combination of different types of failures to test the accuracy of the data after judgement. In the experiment, 12,000 pieces of data are recorded in 1 second. We divided these data into several cases of different failure data, which are read in order to compare and evaluate the results. After doing many tests of the data, the experimental results showed that when the peak value of the same type of failure was taken as " 1 " second, the success rate was very high and the problem was that the modelling was based on a relationship of 1 second. From the experimental results, we can see that the greater the number of data read, the higher the success rate of failure detection as shown in table 3 .

\section{Conclusion}

In this paper, we have proposed a method for the diagnosis and prediction of possible failures in machine tools based on the analysis of vibration data obtained. Our research focuses on analysing the vibration data of bearings. Based on the statistical data on peak coordinates of faults, we propose a model to classify and identify potential failures for each type data obtained. The experimental results show that our method is highly accurate in diagnosing failures and can significantly reduce the maintenance costs, labour costs, and dead time of machine tools.

\section{References}

1. Senthilkumar M, Vikram M, and Pradeep B 2015 Vibration Monitoring for Defect Diagnosis on a Machine Tool: A Comprehensive Case Study India. International Journal of Acounstics and Vibrations 2049

2. Renwick J T, Babson P E 19852015 Vibration analysis a-proven technique as a predictive maintance tool. IEEE Trans. Ind. Appl. 21 (2) 32432

3. Gohar R, Akturk N 1998 Vibrations associated with ball bearings. ImechE 4364

4. Al-Najjar B 2000 Accuracy, effectiveness and improvement of vibration-based maintenance in paper mills: case studies. J. Sound Vib. 229 (2) 389410

5. Orhan S, Akturk N, and Celik V 2006 Vibration monitoring for defect diagnosis of roller element bearings as a predictive maintenance tool: comprehensive case studies. NDT\&E International 39, 293298 